
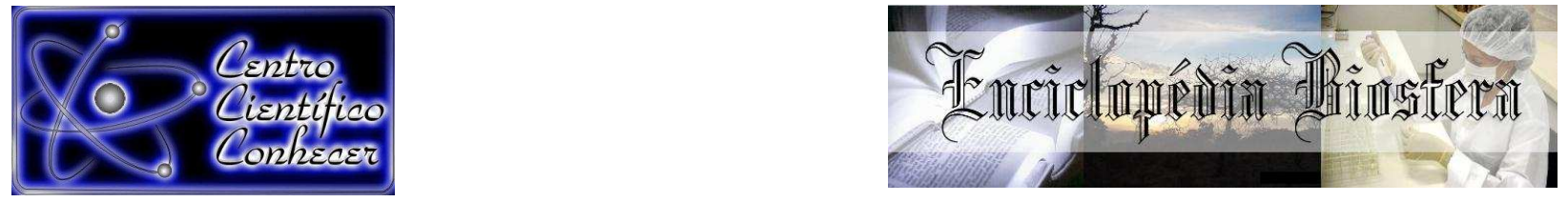

\title{
A OMISSÃO DE INFORMAÇÕES EM PRONTUÁRIOS MÉDICOS DE MULHERES QUE FORAM A ÓBITO POR NEOPLASIAS DO ÚTERO
}

\author{
Maria Eugenia Alcântara Albano ${ }^{1}$, Jéssica Camila Fizinus ${ }^{2}$, Adriana Cunha Vargas \\ Tomaz $^{3}$ \\ ${ }^{1}$ Acadêmica do Curso de Medicina do Centro Universitário de Maringá - \\ UNICESUMAR, Av. Guedner, 1610, Maringá-PR, 87050-900, Brasil, e-mail: \\ maria.eugenia.albano@gmail.com \\ ${ }^{2}$ Acadêmica do Curso de Medicina do Centro Universitário de Maringá - \\ UNICESUMAR, Av. Guedner, 1610, Maringá-PR, 87050-900, Brasil \\ ${ }^{3}$ Docente do Curso de Medicina do Centro Universitário de Maringá - UNICESUMAR, \\ Av. Guedner, 1610, Maringá-PR, 87050-900, Brasil
}

Recebido em: 03/10/2016 - Aprovado em: 21/11/2016 - Publicado em: 05/12/2016 DOI: 10.18677/EnciBio_2016B_126

\begin{abstract}
RESUMO
INTRODUÇÃO: O câncer de útero é o segundo câncer mais prevalente no sexo feminino quando desconsidera-se câncer de pele não melanoma. É uma doença que tem um bom prognóstico quando diagnosticada nas fases iniciais e, para ser diagnosticada precocemente, aspectos essenciais do atendimento à paciente devem ser priorizados e devidamente anotados em prontuários para acompanhamento da evolução clínica. OBJETIVO: O objetivo da pesquisa foi demonstrar a omissão de informações em prontuários de mulheres usuárias do SUS que foram a óbito em Maringá por neoplasias do útero, no período de 2009 a 2014. METODOLOGIA: Pesquisa transversal de cunho epidemiológico. A coleta de dados ocorreu na secretaria de saúde de Maringá a partir das declarações de óbito por neoplasia correspondente ao CID-10: C55, C539 e C541, correspondendo a neoplasia do útero, do colo e do corpo, respectivamente. Na sequência houve a captação do número do SUS e prontuários eletrônicos no sistema gestor. RESULTADOS: Das 40 variáveis analisadas, apenas a data de nascimento, nome da Unidade Básica de Saúde (UBS) e o número do cartão do SUS estavam presentes em todos os prontuários, ao passo que foram ignoradas em $100 \%$ da amostra informações como: etnia, data da menarca, uso de preservativos, realização de atividades físicas, escolaridade, renda familiar e histórico de cauterização. CONCLUSÃO: A pesquisa realizada mostrou que há omissão de dados referente ao exame médico em prontuários, demonstrando de forma indireta a relação da pouca ou nenhuma informação no documento com a evolução ao óbito ocasionado pela doença.
\end{abstract}

PALAVRAS-CHAVE: mortalidade; neoplasias uterinas; registros médicos.

\section{THE OMISSION OF INFORMATION ON MEDICAL RECORDS OF WOMEN WHO DIED OF UTERINE NEOPLASMS}

\section{ABSTRACT}

INTRODUCTION: Uterine neoplasms are the second most prevalent cancer in women, when non-melanoma skin cancer is disregarded. It is a disease that has a ENCICLOPÉDIA BIOSFERA, Centro Científico Conhecer - Goiânia, v.13 n.24; p.1366 2016 
good prognosis when diagnosed in the early stages and to be diagnosed early, essential aspects of the patient care should be prioritized and duly noted in medical records for accompaniment of the clinical course. OBJECTIVE: This research aimed to demonstrate the information omission in medical records belonging to women users of the Unified Health System who died of uterine cancer in Maringá in the period of 2009 to 2014. METHODS: A documentary and epidemiological nature research. The data collection occurred from death certificates of uterine cancer, relative to ICD-10: C55, C539 and C541, corresponding to cancer of the uterus, cervix and body, respectively. Subsequently the SUS number and the electronic medical records were obtained in the manager system. RESULTS: Among the 40 variables analyzed, only the date of birth, UBS name where the service happened and the SUS card number were present in all the records, whereas were ignored in $100 \%$ of the sample information such as: ethnicity, date of menarche, condom use, physical activities, education, family income, and cauterization history. CONCLUSION: The research showed that there is omission of information in medical records concerning the medical exam, demonstrating indirectly the relation between little or no information in the document and the evolution to death caused by the disease.

KEYWORDS: medical records; mortality; uterine neoplasms.

\section{INTRODUÇÃO}

As mulheres estão suscetíveis ao câncer de colo de útero em qualquer idade da vida, sendo mais diagnosticadas entre 35 e 55 anos de idade (INSTITUTO DO HPV, 2013). Na América Latina e Caribe, este é o segundo câncer mais prevalente no sexo feminino quando desconsidera-se câncer de pele não melanoma (GLOBOCAN, 2012).

Dentro de uma série histórica desenvolvida pela IARC (International Agency for Research on Cancer), constatou-se que no período de 1979 a 2005 houve um acréscimo de 0,32 por 100.000 na taxa de mortalidade por câncer de colo de útero de mulheres brasileiras (THULER, 2008). Segundo dados do DATASUS (BRASIL, 2013a) houve um aumento de $16 \%$ na mortalidade por neoplasias uterinas desde 2005. Em 2015, na Região das Américas, foi estimada a incidência de mortalidade por câncer de colo de útero de 38.362 mulheres, no entanto, quando agregados às demais neoplasias uterinas, eleva-se para 52.215 mulheres (GLOBOCAN, 2015). No Brasil a estimativa para 2016 é de 16.340 novos casos (INCA, 2015).

Já o câncer do corpo do útero aparece em sexto lugar, com uma incidência mundial de cerca de 319 mil por ano. As principais acometidas são mulheres na menopausa, principalmente entre 50 e 65 anos. Quando diagnosticado no início do aparecimento, é acompanhado de um bom prognóstico, este fato é evidente quando analisam-se as taxas de mortalidade de países desenvolvidos, que geralmente detectam precocemente o câncer, e nota-se que são relativamente mais baixas (INCA, 2014).

O câncer caracteriza-se por um conjunto de células de um tecido ou órgão que crescem de forma lenta e inofensiva ou, rapidamente, de forma agressiva e desordenada, podendo ou não migrar para outras regiões do corpo. Este denominase neoplasia maligna e aquele, benigna. Quando presente em mucosa ou tecido epitelial nomeia-se carcinoma, e quando em glândulas, adenocarcinoma. As neoplasias do útero enquadram-se nessas classificações (BRASIL, 2013b).

A Federação Internacional de Ginecologia e Obstetrícia classifica o câncer do 
útero em: "l" quando o tumor está limitado à região do corpo do útero; "Il" quando o tumor alcança o colo do útero; "III" quando o tumor ultrapassa os limites uterinos, mas ainda está limitado aos órgãos da região pélvica; e "IV" quando o tumor afetou a bexiga ou o ânus, ou mesmo, atingiu outros órgãos a partir de metástases. Esta classificação possui, ainda, subdivisões que englobam extensão e visibilidade do tumor (MUTCH, 2009).

Em relação ao câncer de colo do útero, que inicia-se com modificações intraepiteliais que, progressivamente tornam-se uma lesão cancerosa invasora, a classificação adotada até os dias atuais é a de Richart, em vigor desde 1967. O critério avaliado são as camadas acometidas pela neoplasia intra-epitelial cervical (NIC). Assim, é dividida em NIC I, NIC II e NIC III, sendo que no grau I observa-se uma desordenação apenas das camadas basais do epitélio estratificado, no grau II essa desordem aparece em três quartos da espessura do epitélio e no grau III, em todo o epitélio. A classificação adotada no Brasil divide as lesões intra-epiteliais escamosas em baixo grau (LSIL) e alto grau (HSIL), sendo que esta corresponde aos NIC II e III, e aquela ao NIC I (BRASIL, 2013b).

No geral, o estágio inicial das neoplasias de útero é assintomático, porém, com a evolução da doença podem aparecer alguns sintomas como corrimento e sangramento vaginal (A.C.CAMARGO CANCER CENTER, 2014). Portanto, o diagnóstico na fase assintomática é o conjunto de uma boa anamnese aliada ao exame citopatológico do colo do útero realizado anualmente e a busca ativa de mulheres em idade fértil (BRASIL, 2013b). Estes garantem o diagnóstico e já é comprovado que, aliado ao tratamento adequado, diminuem a incidência do câncer do colo do útero (INSTITUTO DO HPV, 2013).

Além do exposto acima, é necessário documentar as ações médicas para acompanhamento da evolução clínica da paciente e, para isso, é utilizado o prontuário médico. Este é um documento diretamente relacionado ao tratamento da doença quando corretamente utilizado. Assim, irregularidades no preenchimento desse documento estão relacionadas a danos nocivos e irreparáveis para a saúde do paciente, além disso podem comprometer a atividade profissional do médico ou até mesmo da instituição em que este atua. Portanto, registrar corretamente as informações é dever de todos os profissionais da área de saúde (TONELLO et al., 2013). Diante do apresentado, o objetivo do presente estudo foi demonstrar a omissão de informações em prontuários de mulheres usuárias do SUS que foram a óbito em Maringá por neoplasias do útero, no período de 2009 a 2014.

\section{MATERIAL E MÉTODOS}

Trata-se de uma pesquisa documental, epidemiológico e transversal. Esta engloba mulheres usuárias do Sistema Único de Saúde (SUS) que foram a óbito em Maringá por neoplasias de útero correspondente ao CID 10: C55 Neoplasia maligna do útero, porção não especificada, C539 Neoplasia maligna do colo do útero, não especificado e C541 Neoplasia maligna do corpo do útero, no período de 2009 a 2014. Os critérios de exclusão foram: mulheres que foram a óbito por neoplasias do útero em outros municípios, fora do período compreendido, que apresentaram outras causas de óbito ou que não são usuárias do SUS.

Dados encontrados em livros, DATASUS, cadernos de atenção básica e artigos publicados referentes ao assunto foram relacionados com as variáveis presentes e as variáveis ausentes nos prontuários da Secretaria Municipal de Saúde de Maringá. Estes foram adquiridos por meio de informações da Vigilância Epidemiológica e prontuários eletrônicos coletados no Sistema Gestor, de mulheres 
usuárias do Sistema Único de Saúde da cidade de Maringá e que faleceram por neoplasias uterinas.

Primeiramente foi solicitada junto a vigilância epidemiológica da secretaria municipal de saúde de Maringá a lista de óbitos por neoplasia de útero correspondente ao CID-10: C55, C539 e C541. Posteriormente, neste mesmo local, as mulheres foram identificadas no sistema gestor para captação do número do SUS, e após esta etapa foi realizada a procura dos prontuários eletrônicos. A partir dos dados da vigilância epidemiológica de Maringá constatou-se que um total de 89 mulheres foram a óbito por neoplasias uterinas no período estudado. Dentre as 89, não foram encontrados cinco prontuários. Dos 84 prontuários restantes, 27 estavam em branco, ou seja, não possuíam informações presentes, portanto, a pesquisa foi baseada na análise de 57 prontuários eletrônicos de mulheres que foram a óbito por neoplasias uterinas na cidade de Maringá no período de 2009 a 2014.

$O$ instrumento de coleta de dados utilizado foi dividido em sete categorias contendo informações sobre: identificação da paciente, dados da unidade básica de saúde, antecedentes pessoais e familiares, história da doença atual, histórico de câncer em outro local, tratamento e óbito. Dentro de cada categoria foram analisadas, ainda, algumas variáveis. $\mathrm{Na}$ identificação da paciente foi avaliada a data de nascimento, idade, etnia e o número do cartãoSUS e o número de atendimentos que realizou na Unidade Básica de Saúde desde o primeiro dia até o óbito.

Quanto aos dados da Unidade Básica de Saúde (UBS), identificou-se o nome da UBS que atendeu a paciente. Referente aos antecedentes pessoais e familiares, foram avaliados dados relativos à hipertensão, diabetes, tabagismo, exame citopatológico, data e quantidade de exames citopatológicos, data da menarca, primeira relação sexual, número de parceiros sexuais, uso de anticoncepcional, número de filhos, uso de preservativo, história familiar de câncer, realização de atividade física, estado civil, escolaridade, renda familiar e número de pessoas que vivem com a renda familiar.

Sobre a história da doença atual, foi avaliada a data da primeira queixa ginecológica, identificação da queixa ginecológica, diagnóstico, data do diagnóstico do câncer, a classificação do CID-10, intervalo entre as citologias, satisfatoriedade dos tipos de células, se a paciente realizou cauterização, se a paciente se submeteu a alguma cirurgia, se houve metástase e, se houve, o local da metástase. No histórico de câncer foi avaliado se houve diagnóstico de câncer em outro local e qual o local do câncer anterior. Quanto ao tratamento, foi avaliado a data do início, tipo de tratamento e se houve presença de acompanhante nas consultas. E referente ao óbito, verificou-se a data e causa deste.

A organização, tabulação e sumarização dos dados foram realizadas no Microsoft Excel 2010. Os dados foram analisados por meio do software Epi Info 7.1.5 com estatística descritiva, utilizando frequências absolutas e relativas. As informações dos prontuários foram de acesso apenas das autoras e orientadora do projeto, de maneira que a identidade das pacientes foi preservada.

Os aspectos éticos que envolveram a presente pesquisa são embasados nas Diretrizes e Normas Regulamentadoras de Pesquisas Envolvendo Seres Humanos do Conselho Nacional de Saúde, Resolução CNS 466/2012 (BRASIL, 2013c). Após a liberação para pesquisa na Secretaria Municipal de Saúde, o projeto foi submetido à apreciação do Comitê de Ética em Pesquisa da UNICESUMAR, Centro Universitário Cesumar, com parecer consubstanciado nํ⒈193.439. 


\section{RESULTADOS E DISCUSSÃO}

De acordo com o CONSELHO FEDERAL DE MEDICINA (2002), na Resolução n.ำ 1.638/02, prontuário é "documento único, constituído de um conjunto de informações, sinais e imagens registrados, gerados a partir de fatos, acontecimentos e situações sobre a saúde do paciente e a assistência a ele prestada, de caráter legal, sigiloso e científico, que possibilita a comunicação entre membros da equipe multiprofissional e a continuidade da assistência prestada ao indivíduo". Na presente pesquisa foram analisados 57 prontuários eletrônicos de mulheres que foram a óbito por neoplasias uterinas em Maringá no período de 2009 a 2014.

Em consultas de cunho ginecológico, o prontuário deve seguir a semiologia ginecológica que se baseia em anamnese e exame físico desta especialidade. Com isso, na anamnese é primordial que seja feita a identificação da paciente colhendose nome, data de nascimento, estado civil e profissão (OLIVEIRA et al., 2014). Sobre a identificação da paciente, a Figura 1 mostra que apenas $21 \%$ dos prontuários médicos analisados não apresentavam essas informações, fazendo desta categoria a mais prevalente dentre as analisadas. A data de nascimento, o nome da UBS que realizou o atendimento e o número do cartão do SUS estavam presentes em $100 \%$ dos prontuários. Por outro lado foram ignoradas em todos os prontuários informações referentes à etnia, escolaridade e renda familiar.

Após a identificação, pergunta-se o motivo da consulta e, se houver relato de sintoma, anota-se para, no questionamento da história da doença atual, investigar mais a fundo (OLIVEIRA et al., 2014). Quanto a história da doença atual, variável básica e primordial a ser analisada, houve um déficit de informações em $70,8 \%$ dos prontuários, como indicado pela Figura 1.

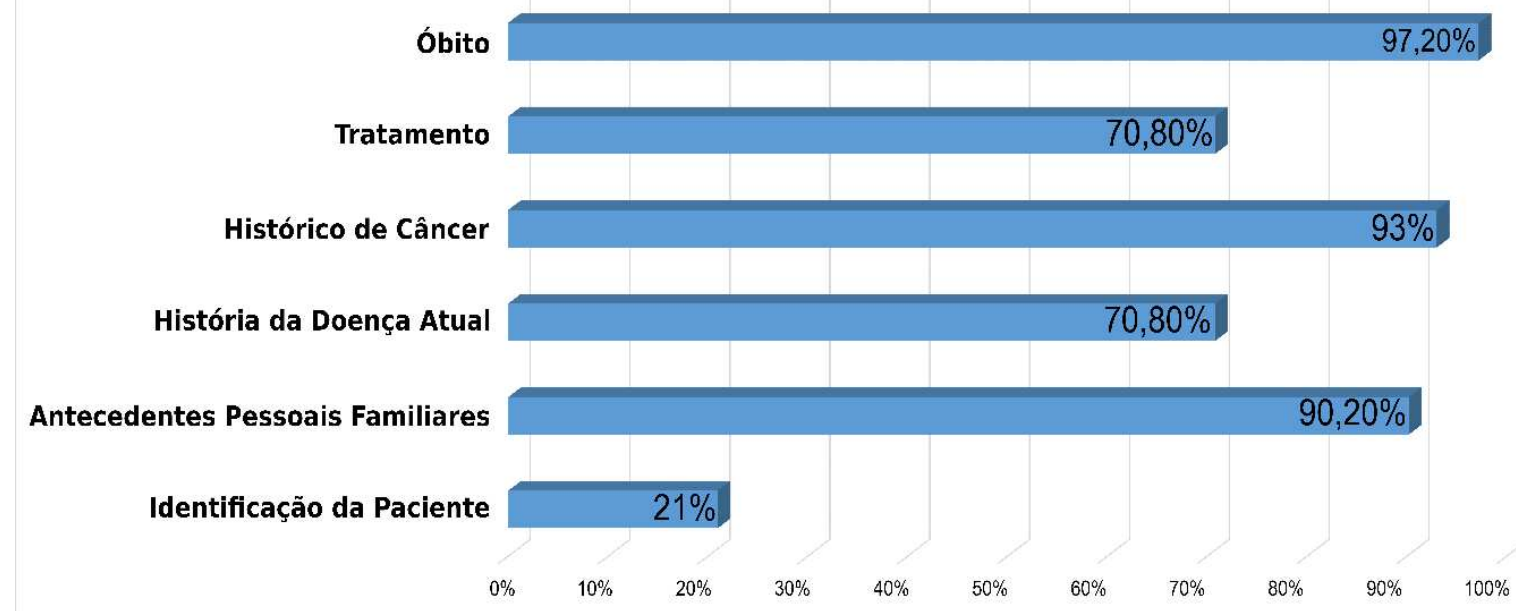

FIGURA 1. Distribuição das informações de prontuários de mulheres que foram a óbito por neoplasias uterinas, referentes às informações ignoradas por grupo de variáveis, Maringá-PR, 2009-2014

Segundo OLIVEIRA et al. (2014), o próximo passo na anamnese é colher informações referentes ao ciclo menstrual da paciente, perguntando a data da menarca, antecedentes e histórico sexual, e a história anticoncepcional. Os dados mais alarmantes apresentados pela pesquisa foram em relação a data da menarca e o uso de preservativo, pois nenhum dos prontuários analisados continham essas informações, sugerindo que não foram investigadas durante a anamnese. Além disso, ANJOS et al. (2013) apontam como fatores de risco para neoplasias do colo ENCICLOPÉDIA BIOSFERA, Centro Científico Conhecer - Goiânia, v.13 n.24; p.1370 2016 
do útero a sexarca precoce, múltiplos parceiros e o raro uso do preservativo. A Tabela 1 indica que $98,2 \%$ dos prontuários analisados não apresentavam dados em relação a idade da primeira relação sexual, 92,3\% não relataram sobre o número de parceiros sexuais, e $91,2 \%$ sobre o uso de anticoncepcional.

$\mathrm{Na}$ Tabela 1 foram reunidos os 11 principais fatores de risco para câncer de útero, sendo eles: hipertensão, diabetes, tabagismo, não realização do exame citopatológico, data da menarca, idade da primeira relação sexual, número de parceiros sexuais, uso de anticoncepcionais, número de filhos, uso de preservativos e história familiar de câncer. Os resultados foram classificados em "ignorado" quando não estavam presentes no prontuário, "sim" quando estavam presentes e "não" quando foram negados ou omitidos pela paciente.

TABELA 1. Distribuição das informações de prontuários de mulheres que foram a óbito por neoplasias uterinas, referentes aos principais fatores de risco desta patologia, Maringá-PR, 2009-2014

\begin{tabular}{lccc}
\hline \multicolumn{1}{c}{ Fator de risco } & Ignorado (\%) & Sim (\%) & Não (\%) \\
\hline Data da menarca & 100 & 0 & 0 \\
Uso do preservativo & 100 & 0 & 0 \\
Idade da sexarca & 98,2 & 0 & 1,8 \\
História familiar de câncer & 93 & 7 & 0 \\
Número de parceiros & 92,3 & 7 & 0 \\
Uso de anticoncepcional & 91,2 & 8,8 & 0 \\
Tabagismo & 87,7 & 7 & 5,3 \\
Diabetes & 82,4 & 14,1 & 3,5 \\
Número de filhos & 80,7 & 19,3 & 0 \\
Não realização do exame & 70,2 & 26,3 & 3,5 \\
citopatológico & & & \\
Hipertensão arterial & 54,4 & 43,9 & 1,7 \\
\hline
\end{tabular}

Seguindo a anamnese investiga-se os antecedentes pessoais e familiares da paciente (OLIVEIRA et al., 2014). Sabe-se que pelo menos 10\% dos casos de câncer de colo de útero tem como fator de risco a hereditariedade, ou seja, a história familiar de câncer (RAFAEL \& MOURA, 2012). Apesar disso, apenas 7\% dos prontuários analisados na presente pesquisa apresentaram relatos sobre o histórico familiar de câncer. Referente aos antecedentes pessoais, no que concerne à periodicidade do exame preventivo, $70,2 \%$ não discorreram sobre a paciente tê-lo realizado ou não, e foram ignorados em todos os prontuários importantes fatores como a realização de atividades físicas e o histórico de cauterizações. De modo geral, em 90,2\% dos 57 prontuários analisados os dados sobre antecedentes pessoais e familiares estavam faltando.

O número de filhos é muito importante como fator de risco, pois as multíparas têm, segundo RAFAEL \& MOURA (2012), um fator de risco para câncer de colo de útero. Por outro lado a AMERICAN CANCER SOCIETY (2015), traz a nuliparidade como fator de risco para o câncer de endométrio, sendo o maior número de filhos, por uma questão hormonal, um fator protetor para esta neoplasia. No entanto, apenas um pouco mais de $19 \%$ dos prontuários analisados de mulheres que foram a óbito por neoplasias uterinas apresentaram informações referentes ao número de filhos das pacientes.

Segundo o Ministério da Saúde (BRASIL, 2013b), existem, ainda, fatores de 
risco para as neoplasias uterinas que são modificáveis como o tabagismo e, para o A.C.CAMARGO CANCER CENTER (2014), a hipertensão e a diabetes mellitus estão diretamente ligadas ao carcinoma de endométrio. A hipertensão foi o fator de risco mais relatado nos 57 prontuários analisados (43,9\%), no entanto $54,4 \%$ dos prontuários ignoraram esta informação. Sobre diabetes e tabagismo, mais de $80 \%$ dos documentos apresentavam déficit de informação quanto a estas comorbidades.

Analisando a média geral de atendimentos por mulher na UBS $(25,1$ atendimentos) verificou-se que os fatores de risco foram negligenciados durante várias consultas da mulher na unidade básica de saúde (tabela 2). Além disso, a quantidade média de consultas nas quais os fatores de risco foram completamente ignorados, equivalem ao número de vezes que estes poderiam ter sido relatados nos prontuários.

TABELA 2. Distribuição média de atendimentos de mulheres que foram a óbito por neoplasias uterinas, referente aos principais fatores de risco. Maringá-PR, 2009-2014

\begin{tabular}{lcc}
\hline \multicolumn{1}{c}{ Fator de risco } & $\begin{array}{c}\text { Presença do fator de risco } \\
\%\end{array}$ & $\begin{array}{c}\text { Fatores ignorados } \\
\%\end{array}$ \\
\hline Número de filhos & 36 & 88 \\
Tabagismo & 67,0 & 49,2 \\
Preservativo & - & 25,15 \\
Menarca & - & 25,1 \\
História familiar & 9 & 24,6 \\
Número de parceiros & 14,5 & 24,4 \\
Primeira relação sexual & - & 23,7 \\
Anticoncepcional & 105 & 23,4 \\
Diabetes & 69,0 & 18,6 \\
Não realização do exame & 88,0 & 12,1 \\
citopatológico & & \\
Hipertensão arterial & 43,7 & 11,9 \\
\hline
\end{tabular}

As mulheres hipertensas tiveram uma média de aproximadamente 44 consultas nas unidades de saúde, ao passo que as pacientes diabéticas e tabagistas tiveram, em média, 69 e 67 atendimentos, respectivamente. Pacientes que não se encontravam em dia quanto a realização do exame citopatológico tiveram uma média de 88 atendimentos nos quais poderiam ter sido melhor orientadas quanto a importância desse exame, e até mesmo tê-lo realizado.

Os fatores de risco menarca precoce e o uso ou não do preservativo, por não constarem em nenhum prontuário, sugere-se que não tiveram chances de serem identificados nas pacientes avaliadas. A data da primeira relação sexual foi ignorada em praticamente todos os prontuários, exceto em um no qual a paciente relatou a inexistência desse fator.

O uso de anticoncepcional oral só estava presente em um dos prontuários, e essa mesma paciente realizou 105 consultas na UBS, no período de 2010 a 2013, o que resulta numa média de 105 atendimentos nos quais poderiam ter sido feitas instruções referentes ao câncer de útero e esse fator de risco. As mulheres multíparas obtiveram uma média de 36 atendimentos em que poderiam ter sido rastreadas para neoplasias uterinas. Por fim, mulheres com uma história familiar de câncer tiveram, em média, nove atendimentos que poderiam ter sido explorados e 
utilizados como base para orientação sobre fatores de risco para câncer de útero.

A pesquisa apresentou limitações devido à falta dos prontuários que não foram encontrados $(5,6 \%$ dos prontuários), bem como os prontuários que foram encontrados, porém que não continham nenhuma informação relatada $(30,3 \%)$, que acabaram por restringir o estudo a apenas 57 prontuários (64\%).

\section{CONCLUSÃO}

Entende-se que a omissão de informações pode refletir na resolução desfavorável do câncer de útero, demonstrando de forma indireta a relação da pouca ou nenhuma informação no documento com a evolução ao óbito ocasionado pela doença. Isso ocorre porque há omissão de informações essenciais à semiologia ginecológica, como queixa ginecológica, data e número de exames citopatológico realizados, história anterior e familiar de câncer, quantidade de parceiros sexuais, uso de preservativos, entre outros dentro das quarenta variáveis analisadas.

Conclui-se que existe omissão de dados referente a anamnese nos prontuários, com isso a frequência da realização do exame citopatológico e o rastreamento de mulheres podem ficar inadequados, resultando em diagnóstico tardio da doença e levando ao prognóstico desfavorável desta. A presente pesquisa alerta sobre a importância da realização de uma boa anamnese, de exames citopatológicos periódicos e do preenchimento adequado dos prontuários. Estes, contendo todos os detalhes clínicos para que, em atendimentos posteriores, a história clínica da paciente seja complementada e resulte no melhor prognóstico possível para cada caso.

\section{REFERÊNCIAS}

A.C.CAMARGO CANCER CENTER. Departamento de Ginecologia. Manual de Condutas em Ginecologia Oncológica. 2. ed. São Paulo, 2014.

\section{AMERICAN CANCER SOCIETY. Endometrial (Uterine) Cancer. 2015. 47p.}

ANJOS, S.J.S.B.; RIBEIRO, S.C.; LESSA, P.R.A.; NICOLAU, A.I.O.; VASCONCELOS, C.T.M.; PINHEIRO, A.K.B. Fatores de risco para o câncer de colo do útero em mulheres reclusas. Revista Brasileira de Enfermagem, Brasília, v. 66, n. 4, p. 508-513, Aug/2013. Disponível em: <http://dx.doi.org/10.1590/S003471672013000400007>. doi: 10.1590/S0034-71672013000400007

BRASIL. Conselho Nacional de Saúde. Diretrizes e normas regulamentadoras de pesquisa envolvendo seres humanos. Resolução n. 466, de 12 de dezembro de 2012. Lex: Diário Oficial da União, Seção I, p.59. Brasília, DF, 13 jun. 2013c.

BRASIL. Ministério da Saúde. Controle dos cânceres do colo do útero e da mama. 2. ed. Brasília, 2013b.

BRASIL. Ministério da Saúde. Sistema de informações sobre mortalidade. Brasília, 2013a. Disponível em: <http://tabnet.datasus.gov.br/cgi/tabcgi.exe?sim/cnv/mat10uf.def> Acesso em: 22 de outubro de 2015.

CONSELHO FEDERAL DE MEDICINA. Define prontuário médico e torna obrigatória a criação da Comissão de Revisão de Prontuários nas instituições de saúde. 
Resolução n. 1.638, de 9 de agosto de 2002. Lex: Diário Oficial da União, Seção I, p.184-5, Brasília, DF, 10 jun. 2002.

GLOBOCAN. Internacional Agency for Research on Cancer. Estimated Cancer Incidence. 2012, 2015.2 Disponível em: <http://globocan.iarc.fr/Pages/fact_sheets_cancer.aspx> Acesso em: 22 de outubro de 2015.

INCA - INSTITUTO NACIONAL DE CÂNCER (Brasil). Estimativa 2014: Incidência de Câncer no Brasil, Rio de Janeiro, 2014, 122p.

INCA - INSTITUTO NACIONAL DE CÂNCER (Brasil). Estimativa 2016: Incidência de Câncer no Brasil, Rio de Janeiro, 2015, 122p.

INSTITUTO NACIONAL DE CIENNCIA E TECNOLOGIA DAS DOENÇAS DO PAPILOMA VÍRUS (São Paulo, SP). Guia do HPV: Entenda de vez os papilomavírus humano, as doenças que causam e o que já é possível fazer para evitá-los, São Paulo, 2013, 41p.

MUTCH, D. The new FIGO staging system for cancers of the vulva, cervix, endometrium and sarcomas. Science Direct, v. 115, n. 3, dez. 2009. Disponível em: <http://dx.doi.org/10.1016/j.ygyno.2009.10.050>. doi: 10.1016/j.ygyno.2009.10.050

OLIVEIRA, J.M.; ALMEIDA, M.C.; ROMÃO, F. Ginecologia, Contracepção e Menopausa: Casos Clínicos. 1.ed. 2014.

RAFAEL, R.M.R.; MOURA, A.T.M.S. Exposição aos fatores de risco do câncer do colo do útero na Estratégia de Saúde da Família de Nova Iguaçu, Rio de Janeiro, Brasil. Caderno de saúde coletiva, Rio de Janeiro, v. 20, n. 4, p. 499-505, 2012. Disponível em: <http://dx.doi.org/10.1590/S1414-462X2012000400014>. doi: 10.1590/S1414-462X2012000400014

THULER, L.C. S. Mortalidade por câncer do colo do útero no Brasil. Revista Brasileira de Ginecologia e Obstetrícia, Rio de Janeiro, v. 30, n. 5, p. 216-218, mai. 2008. Disponível em: <http://dx.doi.org/10.1590/S0100-72032008000500002>. doi: 10.1590/S0100-72032008000500002

TONELLO, I.M.S.; NUNES, R.M.S.; PANARO, A.P. Prontuário do Paciente: A Questão do Sigilo e a Lei De Acesso à Informação, Revista Informação \& Informação: Universidade Estadual de Londrina, v. 18, n. 2, p. 193-210, mai/ago. 2013. Disponível em: $<$ http://dx.doi.org/10.5433/19818920.2013v18n2p193>. doi: 10.5433/1981-8920.2013v18n2p193 